\title{
Évaluation et surveillance des rejets radioactifs des installations nucléaires en Suisse
}

\author{
Ch. MURITH ${ }^{1}$
}

(Manuscrit reçu le 20 mars 2003, accepté le 6 janvier 2004)

RÉSUMÉ L'évaluation et la surveillance des rejets radioactifs des installations nucléaires visent à garantir la protection de la population et de l'environnement vis-à-vis des rayonnements ionisants. Répondre à cette préoccupation à la fois d'ordre réglementaire, scientifique et d'information du public nécessite la mise en ouvre d'un programme de contrôle fíable des rejets, d'une surveillance radiologique vigilante dans l'environnement et d'un système d'information compréhensible du public. L'acceptation des résultats et de leur interprétation par l'opinion requiert que la surveillance des rejets ef l'évaluation de leur impact soient confiées à des organismes accrédités et indépendants des exploitants d"installations nucléaires. C'est le cas en Suisse, où la loi et I'ordonnance de radioprotection stipulent que l'Office fédéral de la santé publique (OFSP) est responsable de ces tâches en collaboration avec les laboratoires cantonaux et les instituts universitaires. Cette publication présente un aperçu des modalités actuelles d'évaluation de l'impact des rejets des installations nucléaires suisses sur l'environnement et la population avoisinante ainsi que du rôle et de l'organisation de la surveillance. Certains enseignements et perspectives dans ce domaine sont également présentés.

ABSTRACT Evaluation and surveillance of radioactive waste of the nuclear installations in Switzerland.

The evaluation and surveillance of nuclear power plants aims to guarantee the protection of the population and of the environment against ionizing radiation. Facing this challenge requires to put in place a liable control program concerning the radioactive releases as well as a thorough radiological surveillance of the environment and a comprehensible information system of the public. In order to obtain public acceptance, the surveillance of the releases and the evaluation of their impact have to be confined to an accredited organism which is independent of the nuclear power industry. Such is the case in Switzerland, where the radioprotection law requires that the federal office of public health (SFOPH) is responsible for this task, in collaboration with local and university laboratories and institutes. This publication presents a overview of the actually used evaluation methods concerning the impact of the radioactive releases from the Swiss nuclear power plants on the local environment and population as well as the role of the surveillance organization. Also presented are certain lessons to be learned and perspectives to be considered in this field.

${ }^{1}$ Oftice fédéral de lá santé publique, 3003 Berne, Suisse. 


\section{Introduction}

Le caractère abstrait, invisible et impalpable de la radioactivité explique en partie l'inquiétude de la population qui en craint la diffusion insidieuse autour des installations nucléaires. Certes, l'impact en terme de dose des rejets des centrales nucléaires suisses sur l'homme reste nettement inférieur à la limite réglementaire et très faible en comparaison de la référence que représente la radioactivité d'origine naturelle. Cependant une information responsable ne doit pour rester crédible ni exagérer, ni minimiser le risque correspondant, compte tenu des limites des connaissances scientifiques, comme celle concernant l'effet des faibles doses sur la santé. En conséquence, la mise en œuvre de dispositions préventives visant à réduire les rejets radioactifs et de programmes de prélèvements et de mesures intégrant des procédures accréditées ainsi que l'utilisation de techniques très sensibles de détection sont justifiées.

\section{Modalités d'évaluation actuelles de l'impact des rejets}

L'indicateur qui sert en Suisse pour évaluer l'impact des rejets des installations nucléaires est la dose qui en résulte pour la population. Compte tenu de la réglementation stricte en matière de rejets et des moyens mis en ouvre pour les maintenir aussi faibles que possible, il n'est pas aisé de déterminer de manière exhaustive leur incidence dans l'environnement. C'est pourquoi les mesures des rejets effectifs à la source avant dilution par voie atmosphérique ou liquide constituent la base de l'évaluation des doses à la population. À partir de ces données, des scénarios d'exposition basés sur les modèles de dispersion des radionucléides et de leur transfert dans les différents compartiments environnementaux permettent de calculer l'jmpact maximal en terme de dose de l'exploitation annuelle d'une installation nucléaire sur une personne hypothétique vivant en permanence dans son voisinage. La figure 1 présente le suivi annuel de cet impact pour les centrales nucléaires suisses. La fiabilité de l'évaluation des doses, dont l'incertitude dépend du réalisme des modèles, est examinée par un ensemble de mesures environnementales permettant de contrôler la validité des modèles, le cas échéant, de mettre en évidence un impact occulté par l'approche calcul à partir des rejets effectifs et de déceler d'éventuels phénomènes d'accumulation à long terme.

\section{Rôle et organisation de la surveillance}

Le rôle de la surveillance de la radioactivité dans l'environnement est de compléter le contrôle réglementaire des émissions par des prélèvements et des mesures susceptibles de suivre le devenir des rejets et d'aider à la compréhension de leur 


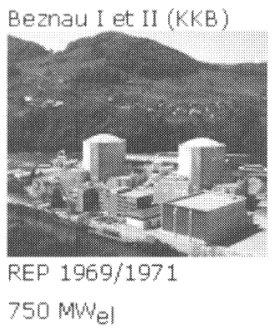

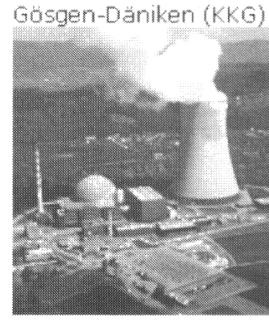

REP 1979,1020 MWel

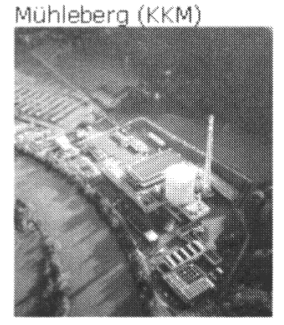

REB $1972,370 \mathrm{MWel}$

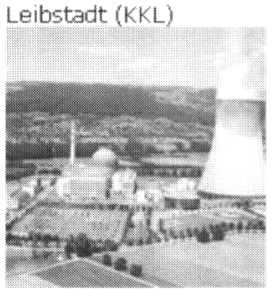

REB $1984,1175 \mathrm{MWel}$

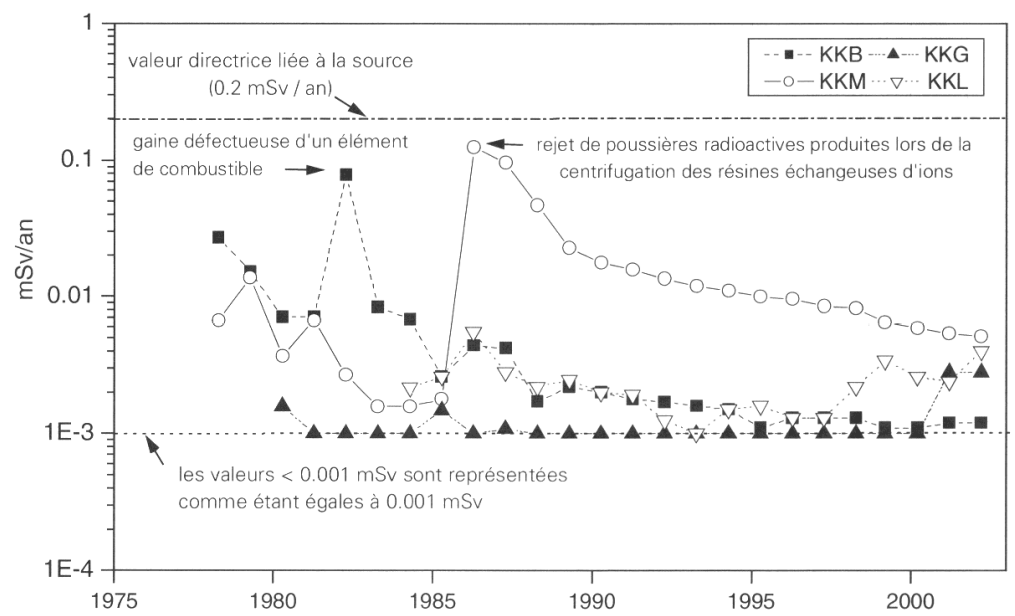

Figure 1 - Dose (calculée) accumulée par la personne (adulte) la plus exposée dans le voisinage de chacune des centrales nucléaires suisses pour chaque année de 1981 à 2002 (Données DSN). La directive $R-41$ de la DSN fixe à $0,2 \mathrm{mSv} / \mathrm{an}$ la dose limite liée à la source. Les valeurs inférieures à $1 \mu \mathrm{S} v / a n$ sont prises égales à $1 \mu \mathrm{Sv} / a n$. La limite légale pour la radioactivité artificielle est $1 \mathrm{mSv} / \mathrm{an}$.

Calculated doses for the most affected persons (adults) in the vicinity of the Swiss nuclear power plants. Data from the Swiss Nuclear Safety Inspectorate (HSK). Its directive R-41 fixes the limit for the dose from the source at $0.2 \mathrm{mSv} / \mathrm{year}$. Values below $1 \mu \mathrm{Sv} / \mathrm{year}$ are plotted equal to $1 \mu \mathrm{Sv}$ /year. The legal dose limit for artificial radioactivity is $1 \mathrm{mSv} / \mathrm{year}$.

dispersion. Pour y parvenir, il est souvent indispensable de dépasser le cadre réglementaire par la planification de mesures de bas niveau et d'études radioécologiques à caractère scientifique. En Suisse, la surveillance des taux de radioactivité dans l'environnement des installations nucléaires intègre les réseaux automatiques de mesure du débit de dose ambiant NADAM et MADUK ainsi que le réseau automatique de surveillance des aérosols de l'air RADAIR et un 
ensemble d'examens systématiques dans les compartiments environnementaux suivants :

- l'air : l'OFSP exploite trois systèmes offrant un compromis entre la rapidité et la sensibilité de détection. Le réseau RADAIR (onze moniteurs $\alpha / \beta$ avec compensation de la radioactivité naturelle, trois moniteurs iode et un de spectrométrie gamma) de faible débit $10 \mathrm{~m}^{3} / \mathrm{h}$, les collecteurs de débit moyen $\left(40 \mathrm{~m}^{3} / \mathrm{h}\right)$ au voisinage des quatre centrales nucléaires suisses et ceux à haut débit $\left(600\right.$ à $\left.800 \mathrm{~m}^{3} / \mathrm{h}\right)$ dans cinq sites ciblés. Les limites de détection de ces systèmes pour le ${ }^{137} \mathrm{Cs}$ sont : $0,5 \mathrm{~Bq} / \mathrm{m}^{3}$ en $1 / 2$ heure avec RADAIR, $2 \mu \mathrm{Bq} / \mathrm{m}^{3}$ avec les collecteurs de débit moyen (échantillons mensuels) et $0,5 \mu \mathrm{Bq} / \mathrm{m}^{3}$ avec les collecteurs haut débit (échantillons hebdomadaires). Les mesures de radionucléides spéciaux $\left({ }^{85} \mathrm{Kr},{ }^{133} \mathrm{Xe},{ }^{7} \mathrm{Be},{ }^{10} \mathrm{Be}\right.$ et $\left.{ }^{210} \mathrm{~Pb}\right)$ à la station du Jungfraujoch $(3580 \mathrm{~m})$ et des aérosols collectés dans la tropopause en collaboration avec l'aviation militaire complètent cette surveillance de l'air ;

- les précipitations : l'OFSP exploite des collecteurs de grande surface $\left(1 \mathrm{~m}^{2}\right)$ et détermine mensuellement les teneurs $\gamma$ et ${ }^{3} \mathrm{H}$ des précipitations. Pour le tritium une surveillance ciblée, incluant l'humidité de l'air, est mise en cuvre auprès des entreprises industrielles utilisant ce radionucléide en particulier dans le secteur horloger ;

- les systèmes aquatiques: les eaux des rivières suisses, des stations d'épuration des eaux usées des grandes agglomérations et de nappes phréatiques sont contrôlées mensuellement du point de vue des radionucléides $\gamma$ et $\mathrm{du}{ }^{3} \mathrm{H}$. Un programme particulier a été initié pour la surveillance des sources d'eau potable avec le Centre d'hydrogéologie de l'université de Neuchâtel et pour la surveillance des rejets du CERN avec l'Institut Forel de l'université de Genève. Le bilan des écoulements liquides des centrales nucléaires est dressé annuellement à partir des analyses de trappes à sédiments. La surveillance est complétée par des mesures réalisées sur la végétation aquatique et les poissons ;

- le milieu terrestre : comme pour l'air, l'OFSP combine les performances de sensibilité et de rapidité de trois méthodes de cartographie du milieu terrestre. L'aéroradiamétrie pour le recensement rapide mais sommaire des zones les plus marquées, la spectrométrie $\gamma$ in situ pour une caractérisation rapide d'un site du point de vue des radionucléides $\gamma$ et de leur contribution individuelle à l'exposition externe du public, et enfin les analyses $\alpha, \beta$ et $\gamma$ différées en laboratoire sur des échantillons ponctuels. Ces dernières analyses portent en priorité sur l'herbe, le sol et les denrées alimentaires (exposition interne). Des mesures dans le corps humain (corps entier, vertèbres humaines, dents) constituent un contrôle de fiabilité des modèles de transfert jusqu'à l'ultime maillon de la chaîne. 
L'organisation de cette surveillance repose sur le laboratoire d'essai de l'OFSP pour la radioactivité de l'environnement, accrédité selon ISO/IEC 17025 pour la spectrométrie gamma in situ et en laboratoire ainsi que pour les analyses tritium et sur la coordination avec les laboratoires accrédités des organismes suivants :

- division principale pour la sécurité des installations nucléaires (DSN) à Villigen : exploitant du réseau de débit de dose (MADUK) au voisinage des centrales nucléaires et du PSI ;

- caisse nationale suisse d'assurances (Suva) à Lucerne: entreprises industrielles;

- centrale nationale d'alarme (CENAL) à Zürich : réseau national du débit de dose (NADAM) ;

- laboratoires cantonaux: surveillance de la radioactivité dans les denrées alimentaires ;

- laboratoire AC de Spiez et Institut Paul Scherrer (PSI) à Würenlingen ;

- institut fédéral pour l'aménagement, l'épuration et la protection des eaux (EAWAG) ;

- universités de Berne, Genève et Neuchâtel, Institut de radiophysique appliquée (IRA) de Lausanne, écoles polytechniques de Lausanne et de Zürich, CERN et laboratoires tiers.

La figure 2 rend compte des outils de base exploités en Suisse pour la surveillance de la radioactivité dans l'environnement des installations nucléaires.

\section{Enseignements et perspectives}

L'organisation de laboratoires, qui intègre ceux des chimistes cantonaux dans le programme de surveillance et d'évaluation de l'impact des installations nucléaires, permet en Suisse de favoriser une information de proximité par les acteurs locaux directement impliqués dans les mesures et la rédaction des rapports annuels de l'OFSP. Cette responsabilité partagée jusqu'à l'information de nos concitoyens est un atout de crédibilité des résultats publiés et de leur appréciation critique. L'accès aussi large que possible à une base de données centralisée regroupant l'ensemble des mesures de la radioactivité de l'environnement doit encore être visé (droit à l'information). En outre, la présence régulière des mesureurs sur le terrain constitue une démarche propice au dialogue avec les résidents. Il apparaît que la population en général a des connaissances très partielles sur la radioactivité, le plus souvent basées sur des informations liées à des événements médiatiques. Dans le voisinage des installations nucléaires fort est de constater que le public local paraît mieux informé. Un effort reste à consentir au niveau de la formation (écoles, collèges, ...). L'utilisation de moyens modernes de communication (Internet) 
NADAM (CENAL): 58 stations de mesure du débit de dose ambiant du réseau national

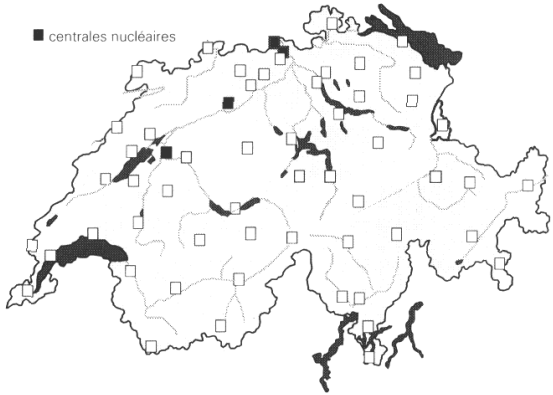

Couverture de l'ensemble du territoire

Réseau RADAIR (OFSP) : 11 stations

de mesures de la radioactivité de l'air

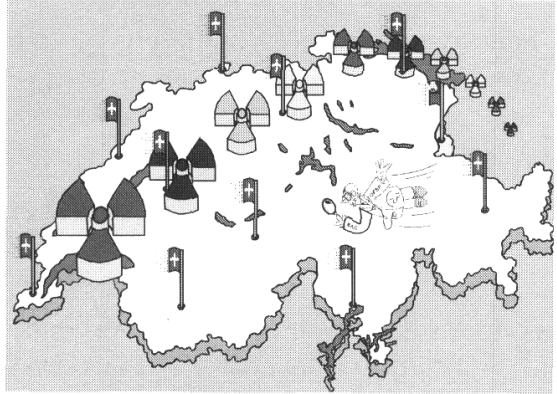

À ce réseau automatique s'ajoutent les collecteurs de débit moyen $\left(40 \mathrm{~Bq} / \mathrm{m}^{3}\right)$ autour des

centrales nucléaires et ceux de haut débit

$\left(600\right.$ à $800 \mathrm{~Bq} / \mathrm{m}^{3}$ ) en 5 points stratégiques

Échantillonnage et mesures $\alpha, \beta, \gamma$ différées

Systèmes aquatiques (EAWAG, Institut Forel...)

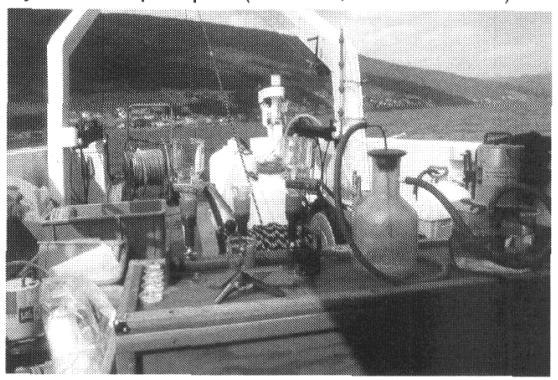

MADUK (DSN) : stations de mesures du débit de dose ambiant en plus des dosimètres TLD

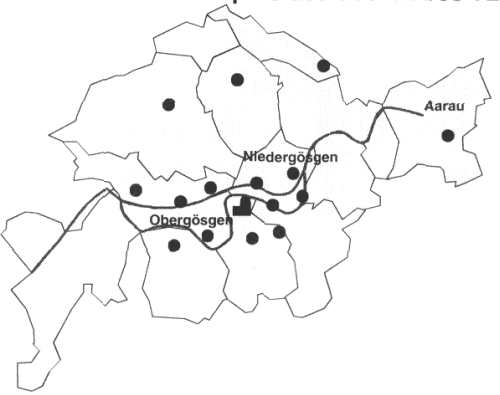

Exemple au voísinage de la CN de Gösgen-Däniken Surveillance in situ par l'OFSP en plus du plan de prélèvements d'échantillons

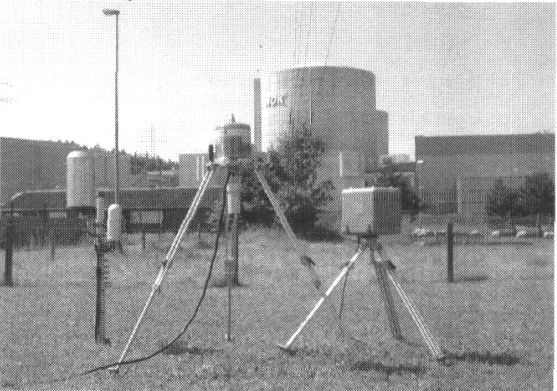

Spectrométrie $\gamma$ in situ avec mesure simultanée du débit de dose ambiant (chambre d'ionisation) à la station météorologique de Beznau.

Mesures selon les recommandations ICRU 53

Autres échantillons environnementaux et humains
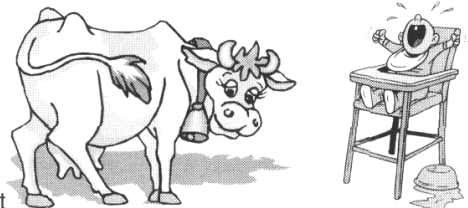

(gamma et strontium)

Dents de lait vertèbres $\left({ }^{90} \mathrm{Sr}\right)$

Laboratoires spécialisés

Denrées alimentaires : chimistes cantonaux

Radionucléides particuliers $\left({ }^{85} \mathrm{Kr},{ }^{14} \mathrm{C} ..\right)$ Uni Berne Mesures corps entier (hôpitaux de Genève et Bâle)

Figure 2 - Outils de surveillance de la radioactivité de l'environnement utilisés en Suisse.

Surveillance tools used in Switzerland for the environmental radioactivity monitoring. 
permet également d'œuvrer pour une information accessible à tous. Une implication des parties prenantes sur la question du démantèlement des installations et de la gestion des déchets semble constituer une priorité de réponse aux préoccupations du public et des générations futures. Leur intégration dans le débat des questions de radioprotection doit être favorisé. Une stratégie de surveillance simultanée et localisée de tous les compartiments de l'environnement est en outre souhaitable afin de corréler plus efficacement les résultats des mesures, de comprendre les processus et d'analyser les causes et les conséquences d'une valeur hors norme plutôt que de se limiter à juxtaposer des valeurs de mesure isolées. Enfin la surveillance devrait comprendre une composante non-permanente pour analyser des problèmes ciblés comme par exemple NORM (Naturally Occuring Radioactive Material) lors de grands chantiers ou la gestion des héritages radioactifs (en particulier radium utilisé dans l'industrie horlogère), favorisant une flexibilité de la surveillance et une dynamique de recherche qui fait parfois défaut dans un programme de routine.

\section{Conclusions}

En Suisse, la surveillance radiologique et l'évaluation de l'impact des rejets des installations nucléaires vise à garantir la protection de la population et de l'environnement vis-à-vis des risques des rayonnements ionisants. La pertinence des modalités de surveillance et de l'évaluation des doses de rayonnements de la population toutes sources confondues est examinée par la Commission fédérale de protection contre les radiations et de surveillance de la radioactivité (CPR), qui publie annuellement une prise de position à l'intention du Conseil fédéral sur la situation de la radioprotection dans notre pays et en informe la presse par communiqué. Cette commission extraparlementaire est indépendante de l'administration. Ses membres ont été choisis en tant qu'experts et non comme représentants de groupes professionnels. La CPR regroupe la sous-commission médicale, la sous-commission environnement et le groupe d'experts pour la dosimétrie en radioprotection. Les compétences ainsi rassemblées recouvrent l'ensemble des domaines d'utilisation des radiations ionisantes et permet à la commission de donner un avis critique et d'émettre des recommandations sur les activités des autorités. Ainsi, la CPR a publié en janvier $200 \dot{4}$ une série de recommandations à l'intention des autorités de surveillance visant à améliorer d'une part l'organisation et la qualité des mesures et d'autre part les modalités de surveillance. Dans son rôle de communication en radioprotection, la CPR entend rester vigilante sur l'ensemble des questions touchant à la protection des personnes et de l'environnement en informant la population de manière compétente et indépendante chaque fois qu'un besoin s'en fera sentir (www.ksr-cpr.ch). 
Ch. MURITH

\section{Pour en savoir plus}

Office fédéral de la santé publique : www.admin.ch/bag.

Centrale nationale d'alarme : www.naz.ch.

Division principale de la sécurité des installations nucléaires : www hsk.psi.ch.

Centrales nucléaires: www.kkg.ch, www.kkl.ch, www.nok.ch, www.bkw.ch.

CERN : www.cern.ch.

Autres : www.eawag.ch, www.hospvd.ch/public/instituis/ira, www. vbs.admin.ch/acls.

CEDRA : www nagra.ch. 